\title{
Quasiparticle Energy Spectrum and Josephson Current in Superconducting SNINS Junctions
}

\author{
William J. Herrera, J. Virgilio Niño, and J. Jairo Giraldo \\ Departamento de Física, \\ Universidad Nacional de Colombia, Bogotá- Colombia.
}

Received on 8 December, 2006

\begin{abstract}
Through the solution of the Bogoliubov de Gennes equations we analyze the effect of different symmetries of the pair potential on the quasiparticle energy spectrum in SNINS junctions (S: superconductor, N: normal metal and I: Insulator). We find that the energy levels are strongly affected by the symmetry of the pair potential, the width of each normal metal ( $a$ and $b$ ) and the strength of the insulating barrier. The energy levels equation generalizes previous results in SNS and SIS junctions. The energy dispersion relation depends on the phase difference of the pair potential. The Josephson current is related to the Andreev levels; when $a=b \simeq \xi_{0}$ and $T<<T_{c}$ this current is approximately $1 / 2$ of the Josephson current transported in an SIS junctions. In general, we find that for $d_{x y}$ symmetries there is always a zero energy state independent of the value of $Z, a$ and $b$.
\end{abstract}

Keywords: Josephson effect; Superconductivity; Andreev levels

\section{INTRODUCTION}

The symmetry of the pair potential is one of the most studied aspects in high critical temperature superconductors. It plays an important role in different transport properties [1]. Inhomogeneities of the order parameter or pair potential $\Delta$ lead to scattering of electrons into holes and vice versa (Andreev reflections)[2]. The Andreev reflections have been used in isotropic and anisotropic superconductors to explain transport properties mainly in NIS, SIS and SNS junctions [3]-[6]. When a normal metal is placed between two superconductors, bound states with $|E|<<|\Delta|$ are formed by multiple Andreev reflections in the $\mathrm{SN}$ and NS interfaces. The energy levels of these bound states are called Andreev Levels. They are important to explain the Josephson effect in these junctions [7] and the quasiparticles behavior in the core of the vortices [8]. The Andreev levels have been determined in SNS anisotropic junctions [9], [10] and the Josephson effect has been studied in SsNSd [11] ( s: s-symmetry, d: d-symmetry ) and SIS [12] junctions. The study of the bound states and the Josephson effect in SINS and SNINS junctions have been only carried out in isotropic superconductors [13]. A general equation for the quasiparticles energy spectrum is found for SNINS junctions. It is applied to SNS, SIS, INS and SNINS junctions, and to study dc Josephson effect in symmetrical SNINS junctions.

\section{THEORY}

The elementary excitations or quasiparticles in a superconductor are described by the Bogoliubov de Gennes (BdG) equations, which can be generalized for anisotropic superconductors [14]. For steady states these equations are

$$
\begin{array}{r}
H_{e}\left(\mathbf{r}_{1}\right) \tilde{u}\left(\mathbf{r}_{1}\right)+\int d \mathbf{r}_{2} \tilde{\Delta}\left(\mathbf{r}_{1}, \mathbf{r}_{2}\right) \tilde{v}\left(\mathbf{r}_{2}\right)=E \tilde{u}\left(\mathbf{r}_{1}\right), \\
-H_{e}^{*}\left(\mathbf{r}_{1}\right) \tilde{v}\left(\mathbf{r}_{1}\right)+\int d \mathbf{r}_{2} \tilde{\Delta}^{*}\left(\mathbf{r}_{1}, \mathbf{r}_{2}\right) \tilde{u}\left(\mathbf{r}_{2}\right)=E \tilde{v}\left(\mathbf{r}_{1}\right),
\end{array}
$$

where $H_{e}\left(\mathbf{r}_{1}\right)=-\hbar^{2} \nabla^{2} / 2 m+V\left(\mathbf{r}_{1}\right)-\mu$ is an electronic hamiltonian and $\mu$ the chemical potential. $\tilde{\Delta}\left(\mathbf{r}_{1}, \mathbf{r}_{2}\right)$ is the pair potential , $\tilde{u}\left(\mathbf{r}_{1}\right)$ and $\tilde{v}\left(\mathbf{r}_{1}\right)$ are the wave functions for the electronand hole-like components of a quasiparticle. The pair potential $\tilde{\Delta}\left(\mathbf{r}_{1}, \mathbf{r}_{2}\right)$ is a function of the position coordinates $\mathbf{r}_{1}$ and $\mathbf{r}_{2}$, and can be transformed to $\bar{\Delta}(\mathbf{R}, \mathbf{r})=\tilde{\Delta}\left(\mathbf{r}_{1}, \mathbf{r}_{2}\right)$, with $\mathbf{R}=\mathbf{r}_{1}-\mathbf{r}_{2}$ and $\mathbf{r}=\left(\mathbf{r}_{1}+\mathbf{r}_{2}\right) / 2$. The Fourier transform of $\bar{\Delta}(\mathbf{R}, \mathbf{r})$ is

$$
\Delta_{F T}(\mathbf{k}, \mathbf{r})=\int d \mathbf{R} e^{-i \mathbf{k} \cdot R} \bar{\Delta}(\mathbf{R}, \mathbf{r}) .
$$

Using the quasiclassical approximation [14], the pair potential $\Delta_{F T}(\mathbf{k}, \mathbf{r})$ is aproximated by, $\Delta_{F T}(\mathbf{k}, \mathbf{r})=\Delta(\hat{\mathbf{k}}, \mathbf{r})$, where $\hat{\mathbf{k}}=\mathbf{k} /|\mathbf{k}|$ is a unit wavevector. Using $\Delta(\hat{\mathbf{k}}, \mathbf{r})$, the BdG equations are

$$
\begin{aligned}
H_{e}(\mathbf{r}) u_{\mathbf{k}}(\mathbf{r})+\Delta(\hat{\mathbf{k}}, \mathbf{r}) v_{\mathbf{k}}(\mathbf{r}) & =E u_{\mathbf{k}}(\mathbf{r}), \\
-H_{e}^{*}\left(\mathbf{r}_{1}\right) v_{\mathbf{k}}(\mathbf{r})+\Delta^{*}(\hat{\mathbf{k}}, \mathbf{r}) u_{\mathbf{k}}(\mathbf{r}) & =E v_{\mathbf{k}}(\mathbf{r}) .
\end{aligned}
$$

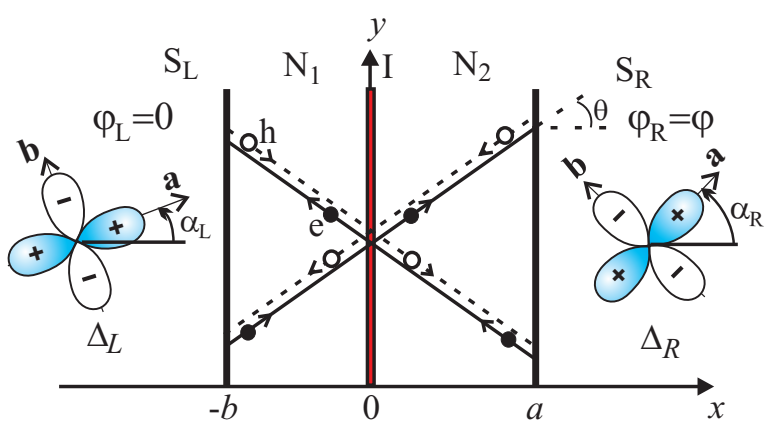

FIG. 1: SNINS junction. The thickness of the normal region is $a+b$, the superconducting regions are semi-infinite and occupy the space defined by $x<-b\left(\Delta_{L}\right)$ and $x>a\left(\Delta_{R}\right)$. The insulating barrier is located in $x=0$ and is modeled by a delta function. For d-symmetry, the pair potential is modeled as $\Delta_{L}(\theta)=\Delta_{0} \cos \left(2 \theta-2 \alpha_{L}\right), \Delta_{R}(\theta)=$ $e^{i \varphi} \Delta_{0} \cos \left(2 \theta-2 \alpha_{R}\right)$, where $\varphi$ is the a global phase difference between the two superconducting regions. The solid and dashed lines represent the electron and the hole-like components of a quasiparticle, respectively.

In the rest of the paper we concentrate on cuprate superconductor junctions. It is suppossed that the quasiparticle 
moves on the $\mathrm{CuO}_{2}$ plane with the $a$ and $b$ axes in the $x-y$ plane; the interfaces are normal to $x$ axis, as indicated in Fig. 1. The insulating barrier is modeled by a delta function, $V(x)=U_{0} \delta(x)$ and the pair potential by $\Delta^{*}(\hat{\mathbf{k}}, \mathbf{r})=$ $\Theta(-b-x) \Delta_{L}(\hat{\mathbf{k}})+\Theta(x-a) \Delta_{R}(\hat{\mathbf{k}})$, where $\Theta(x)$ is the Heaviside function and $\Delta_{L(R)}$ is the pair potential of the left (right) superconductor region. The solutions of the $\mathrm{BdG}$ equations for $\psi(x) \quad\left(\psi(x, y)=\psi(x) e^{i k_{y} y}\right)$ in the $\mathrm{S}_{L}, \mathrm{~N}_{I}, \mathrm{~N}_{I I}$ and $\mathrm{S}_{R}$ regions are respectively,

$$
\begin{aligned}
\psi_{S_{L}}(x)= & C_{-}^{+}\left(\begin{array}{c}
u_{0}^{L,-} \\
v_{0}^{L,-} e^{-i \varphi_{-}^{L}}
\end{array}\right) e^{-i k_{-}^{L,+} x} \\
& +C_{+}^{-}\left(\begin{array}{c}
v_{0}^{L,+} \\
u_{0}^{L,+} e^{-i \varphi_{+}^{L}}
\end{array}\right) e^{i k_{+}^{L,-} x} \\
\Psi_{N_{I}}(x)= & \left(\begin{array}{l}
1 \\
0
\end{array}\right)\left(U_{1} e^{i k_{1}^{+} x}+U_{2} e^{-i k_{1}^{+} x}\right) \\
& +\left(\begin{array}{c}
0 \\
1
\end{array}\right)\left(V_{1} e^{i k_{1}^{-} x}+V_{2} e^{-i k_{1}^{-} x}\right) \\
\psi_{N_{I I}}(x)= & \left(\begin{array}{c}
1 \\
0
\end{array}\right)\left(U_{3} e^{i k_{1}^{+} x}+U_{4} e^{-i k_{1}^{+} x}\right) \\
& +\left(\begin{array}{c}
0 \\
1
\end{array}\right)\left(V_{3} e^{i k_{1}^{-} x}+V_{4} e^{-i k_{1}^{-} x}\right) \\
\Psi_{S_{R}}(x)= & C_{+}^{+}\left(\begin{array}{c}
u_{0}^{R,+} \\
v_{0}^{R,+} e^{-i \varphi_{+}^{R}}
\end{array}\right) e^{i k_{+}^{R,+} x} \\
& +C_{-}^{-}\left(\begin{array}{c}
v_{0}^{R,-} \\
u_{0}^{R,-} e^{-i \varphi_{-}^{R}}
\end{array}\right) e^{-i k_{-}^{R,-} x},
\end{aligned}
$$

with

$$
\begin{aligned}
& u_{0}^{\beta, \pm}=\sqrt{\frac{1}{2}\left[1+i \frac{\Omega_{ \pm}^{\beta}}{E}\right]}, v_{0}^{ \pm}=\sqrt{\frac{1}{2}\left[1-i \frac{\Omega_{ \pm}^{\beta}}{E}\right]}, \\
& k_{ \pm}^{\beta, \varepsilon}=\sqrt{k_{0 x F}^{2}+i \varepsilon \frac{2 m}{\hbar^{2}} \Omega_{ \pm}^{\beta}}, \quad k_{0 x F}=\sqrt{k_{F}^{2}-k_{y}^{2}}, \\
& \Omega_{ \pm}^{\beta}=\sqrt{\left|\Delta_{ \pm}^{\beta}\right|^{2}-E^{2}, k^{ \pm}}=\sqrt{k_{0 x F}^{2} \pm \frac{2 m E}{\hbar^{2}}}, \varepsilon= \pm,
\end{aligned}
$$

where $\beta$ denotes $R$ or $L, k^{ \pm}$are the wave numbers of the electrons (+) and holes (-) in the normal regions, $k_{ \pm}^{\beta, \varepsilon}$ are the wave numbers of the quasiparticles that move in the potentials $\Delta_{L}^{ \pm}$ in the left superconducting region and in the potentials $\Delta_{R}^{ \pm}$in the right superconducting region. These potentials are

$$
\Delta_{ \pm}^{\beta}=\Delta\left( \pm k_{ \pm}^{\beta, \varepsilon}+k_{y} \hat{\mathbf{j}}\right) \equiv\left|\Delta_{ \pm}^{\beta}\right| e^{i \varphi_{ \pm}},
$$

where $\varphi_{+}^{\beta}$ and $\varphi_{-}^{\beta}$ are the phases of the effective pair potentials $\Delta_{+}$and $\Delta_{-}$respectively. For $d$-symmetry $\Delta_{ \pm}^{\beta}=\Delta_{0} \cos (2(\theta \mp$ $\left.\alpha_{\beta}\right)$ ), where $\alpha_{\beta}$ is the angle between the (100) axis of the superconductor and the normal to the interface (cf. fig. 1).

\section{ENERGY SPECTRUM}

From the solution Eq. (4) and the boundary conditions for $\psi(x)$ in $x=-b, x=0$ and $x=a$ we find that the bound states energy levels equation is

$$
F_{+}(E) F_{-}(E)+Z^{2} M_{R} M_{L}=0
$$

with

$$
\begin{aligned}
F_{ \pm} & =\Gamma_{ \pm}^{R} \Gamma_{ \pm}^{L}-e^{i r d} e^{ \pm i\left(\gamma_{ \pm}+\varphi\right)}, M_{R}=\Gamma_{+}^{R} \Gamma_{-}^{R}-e^{-2 i r\left(d-x_{0}\right)} e^{i \gamma_{R}} \\
M_{L} & =\Gamma_{+}^{L} \Gamma_{-}^{L}-e^{-2 i r\left(d+x_{0}\right)} e^{-i \gamma_{R}}, \gamma_{\beta}=\varphi_{+}^{\beta}-\varphi_{-}^{\beta} \\
\gamma_{ \pm} & =\varphi_{ \pm}^{R}-\varphi_{ \pm}^{L}, r=\frac{k_{F}}{\cos \theta} \frac{E}{E_{F}}, d=a+b \\
x_{0} & =\frac{b-a}{2}, \Gamma_{ \pm}^{\beta}=\frac{E-i \Omega_{ \pm}^{\beta}}{\left|\Delta_{ \pm}^{\beta}\right|}, Z=\frac{k_{F} U_{0}}{2 E_{F} \cos \theta} .
\end{aligned}
$$

$Z$ is the strength of the insulating barrier. Equation (8) allows us to find the energy spectrum for any value of $Z$ and $a$, as well as for several symmetries of the pair potentials $\Delta_{L}$ and $\Delta_{R}$. The general solution of this equation is rather difficult. We consider first some special cases, which permit to obtain easily the energy spectrum of SNS, SIS, INS and SNINS systems.

SNS junctions. We obtain from equation (7) for $\mathrm{Z}=0$ : $F_{+}(E) F_{-}(E)=0$. Two sets of energy levels $E_{+}$and $E_{-}$are found, which correspond to particles with wave number $\mathrm{k}_{x}$ positive or negative respectively. The energy levels satisfy the equations

$$
\Gamma_{ \pm}^{R} \Gamma_{ \pm}^{L}=e^{-i r d} e^{ \pm i\left(\varphi_{ \pm}^{R}-\varphi_{ \pm}^{L}+\varphi\right)} .
$$

This spectrum is formed by the Andreev reflections that occur in the SN and NS interfaces.The $E_{+}$and $E_{-}$levels are equivalent to the levels of an $\mathrm{S}_{L+} \mathrm{NS}_{R+}$ and $\mathrm{S}_{L-} \mathrm{NS}_{R-}$ junctions respectively, where the subscript $L_{ \pm}$or $R_{ \pm}$denote the superconductor region with pair potential $\Delta_{ \pm}^{L}$ or $\Delta_{ \pm}^{R}$ respectively. For $E<<\left|\Delta_{ \pm}^{L(R)}\right|$ we find that the two sets of energy levels are given by

$$
E_{n, \pm}=E_{0}\left(2 n+1 \pm\left(\left(\varphi_{ \pm}^{R}-\varphi_{ \pm}^{L}+\varphi\right) / \pi\right)\right.
$$

with

$$
E_{0}=E_{F} \pi \cos \theta / d \text {. }
$$

INS interfaces. If $Z>>0$ equation (7) is reduced to $M_{R} M_{L}=0$; when $M_{L}=0$, we obtain

$$
\Gamma_{+}^{L} \Gamma_{-}^{L}=e^{-2 i r b} e^{ \pm i\left(\varphi_{+}^{L}-\varphi_{-}^{L}\right)}
$$

Comparing this equation with (9), we see that the spectrum is equivalent to one of a junction $\mathrm{S}_{L_{+}} \mathrm{NS}_{L_{-}}$with thickness $2 b$ and pair potential $\Delta_{+}^{L}$ and $\Delta_{-}^{L}$, our result agree with Ref. [12] for surface states, where ZES (zero energy states) appear for $\varphi_{+}^{L}-\varphi_{-}^{L}=\pi$.

SINS junctions. In this section we analyze the spectrum of an SINS junction. This is obtained when $b=0$. For $E<<$ $\left|\Delta_{ \pm}^{L(R)}\right|$ it is possible to find analytic solutions of equation (7), which can be written as

$$
\begin{aligned}
& \cos \left(\frac{E \pi}{E_{0}}\right)\left(\cos \frac{\gamma_{+}-\gamma_{-}}{2}+\left(1-T_{N}\right) \cos \frac{\gamma_{R}+\gamma_{L}}{2}\right) \\
& +\sin \left(\frac{E \pi}{E_{0}}\right)\left(\sin \frac{\gamma_{+}-\gamma_{-}}{2}+\left(1-T_{N}\right) \sin \frac{\gamma_{R}+\gamma_{L}}{2}\right) \\
& +T_{N} \cos \frac{\gamma_{+}+\gamma_{-}+2 \varphi}{2}=0
\end{aligned}
$$


where $E_{0}$ is given by (11) with $d=a$ and $T_{N}=1 /\left(1+Z^{2}\right)$ is the transparence of the junction. We show in table $I$ the energy spectrum for several symmetries of the pair potential. For $s$ symmetries the energy spectrum depends on $Z$ and on the phase $\varphi$ and agrees with previous results [15]. For these junctions the energy spectrum is proportional to the quantum number $n$, as in an SNS junction. The fundamental difference between SINS and SNS junctions is the $\varphi$ dependence of the energy levels. We note that for the symmetries shown in Tab. I, (c) and (d) cases, the epectrum is completely independent of $T_{N}$, as well as in (b) and (c) cases if $\varphi=0$. In figure 2 it is shown the behavior of the energy spectrum with $\varphi$ for $n=0$, for an $\operatorname{SsINS}_{s}$ junction the energy is 0 only for $\varphi=0$ and $T_{N}=1$ while that for an $\mathrm{S}_{s} \mathrm{INS}_{d x y}$ or $\mathrm{S}_{d x 2 y 2} \mathrm{INS} d x y$ junction the energy is 0 for $\varphi=0, \pi$ for any value of $T_{N}$. This shows that the ZES are independent of the strenght of the insulating barrier in these junctions. On the other hand, if the insulating barrier is very strong $T_{N}<<1(Z>>0)$ the energy is independent of the phase $\varphi$ because we have two isolated systems (SI and INS).

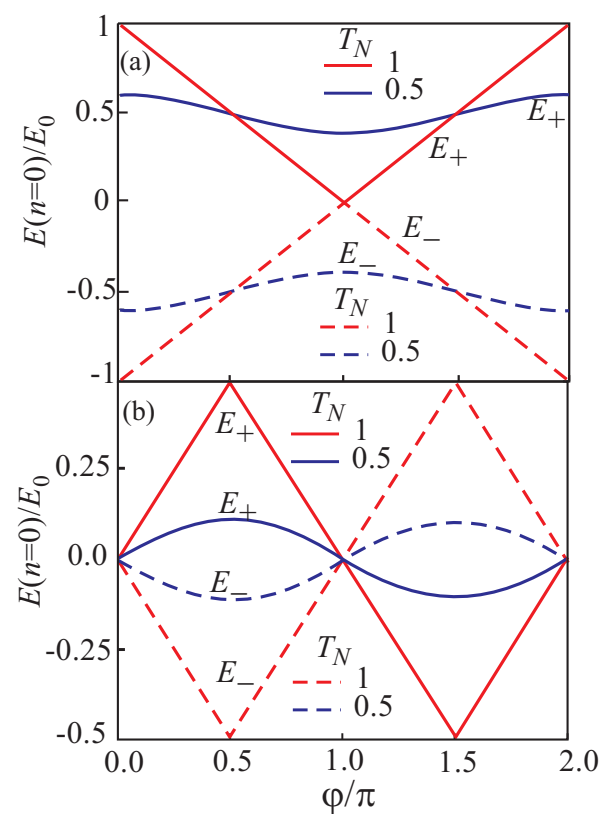

FIG. 2: Energy spectrum for $n=0$ in an SINS junction as a function of $\varphi$. (a) $\mathrm{S}_{S} \mathrm{INS}_{s}$ junction (b) $\mathrm{S}_{d_{x^{2}-y^{2}}} \mathrm{INS}_{d_{x y}}$ junction.

TABLE I: Andreev levels of an SINS junction for $E<<\left|\Delta_{ \pm}^{L(R)}\right|$. The symmetry of each superconducting region is $s$ or $d$.

\begin{tabular}{|c|c|c|}
\hline Junction & $E_{n, \pm} / E_{0}$ & case \\
\hline $\mathrm{S}_{S} \mathrm{INS}_{s}$ & $2 n \pm 1 \mp \frac{1}{\pi} \cos ^{-1}\left(\frac{T_{N}}{2-T_{N}} \cos \varphi\right)$ & (a) \\
\hline $\mathrm{S}_{d_{x 2-y^{2}}} \mathrm{INS}_{d_{x y}}$ & $2 n \pm \frac{1}{\pi} \sin ^{-1}\left(\frac{T_{N}}{2-T_{N}} \sin \varphi\right)$ & (b) \\
\hline $\mathrm{S}_{d_{x y}} \mathrm{INS}_{d_{x y}}$ & $2 n \pm 1 \mp \frac{1}{\pi} \cos ^{-1}(\cos \varphi)$ & (c) \\
\hline $\mathrm{S}_{d_{x y}} \mathrm{INS}_{d_{x^{2}-y^{2}}}$ & $2 n \mp \frac{1}{\pi} \sin ^{-1}(\cos \varphi)$ & (d) \\
\hline
\end{tabular}

SNINS junctions. In the general case the solutions of Eq. (7) are obtained from

$$
\begin{aligned}
& \cos \left(\frac{E \pi}{E_{0}}-\frac{\beta_{1}}{2}\right)+\left(1-T_{N}\right) \cos \left(\frac{E \pi}{E_{0}} \frac{2 x_{0}}{d}+\frac{\beta_{2}}{2}\right) \\
& +T_{N} \cos \left(\frac{\beta_{3}}{2}+\varphi\right)=0
\end{aligned}
$$

with

$$
\begin{aligned}
& \beta_{1}=\gamma_{+}-\gamma_{-}-\left(\phi_{-}^{R}+\phi_{+}^{R}+\phi_{+}^{L}+\phi_{-}^{L}+2 \pi\right) \\
& \beta_{2}=\gamma_{+}+\gamma_{-}+\phi_{-}^{R}-\phi_{+}^{R}+\phi_{-}^{L}-\phi_{+}^{L} \\
& \beta_{3}=\gamma_{R}+\gamma_{L}+\phi_{-}^{L}+\phi_{+}^{L}-\phi_{-}^{R}-\phi_{+}^{R} \\
& \tan \phi_{ \pm}^{\beta}=-\Omega_{ \pm}^{\beta} /|E|, \beta=R, L .
\end{aligned}
$$

The two superconductor regions are correlated by the phase $\varphi$, factor $T_{N} \cos \left(\beta_{3} / 2+\varphi\right)$ in Eq. (14). If $T_{N}=0$ the two regions are isolated and the spectrum is independent of $\varphi$. Equation (14) is completely general and allows us to find the energy spectrum of a SNINS junction for any value of $a, b$, and $T_{N}$, and different symmetries of the pair potential of each superconductor region. Now we consider the case of a symmetrical junction with $a=b=d / 2$ and symmetry $d_{x y}$ in both superconductors. Equation (14) can be written as

$$
\cos (r d+2 \phi)=2 T_{N} \cos ^{2}\left(\frac{\varphi}{2}\right)-1, \phi=\cos ^{-1}\left(\frac{E}{|\Delta|}\right) .
$$

For $d=0$ we get the spectrum of SIS junction

$$
\varepsilon_{ \pm}= \pm \varepsilon, \varepsilon=|\Delta| \sqrt{T_{N}}|\cos (\varphi / 2)| \text {. }
$$

For a thickness $d \sim \xi_{0}$, Eq. (16) can be solved approximately and two energy levels are found

with

$$
E_{ \pm}= \pm \eta \varepsilon
$$

$$
\eta=\sqrt{\frac{4+\delta^{2}-\delta \sqrt{16\left(1-\varepsilon^{2}\right)+\delta^{2}}}{2\left(2+\left(2 \varepsilon^{2}-1\right) \delta^{2}\right)}}, \delta=\frac{2 d}{\pi \xi_{0} \cos \theta} .
$$

For $T_{N}<<1, \eta$ only depends in $d$ and the effect of the normal region is to decrease the value of the energy levels, as is shown in Fig. 3(a)

\section{JOSEPHSON CURRENT}

The Josephson current transported by quasiparticles in the Andreev levels is given by

$$
I_{x}=\frac{2 e}{\hbar} \sum_{n}\left\langle\frac{d E}{d \varphi} f\left(E_{n}\right)\right\rangle,
$$

where $\langle\ldots\rangle$ denotes integration over the angle $\theta$ and $f\left(E_{n}\right)$ is the Fermi-Dirac distribution. Using Eq. (18) $I_{x}$ at temperature $T$ is

$$
\begin{aligned}
I_{x}= & \frac{2 e}{\hbar} \sin \frac{\varphi}{2} \operatorname{sgn}\left(\cos \frac{\varphi}{2}\right) \times \\
& \left\langle\sqrt{T_{N}} \eta|\Delta| \tanh \frac{\sqrt{T_{N}} \eta|\Delta||\cos (\varphi / 2)|}{2 k_{B} T}\right\rangle .
\end{aligned}
$$

At $T=0$ this current is carried by quasiparticles with energy $E_{-}$and is given by

$$
I_{x}=\sin \frac{\varphi}{2} \operatorname{sgn}\left(\cos \frac{\varphi}{2}\right) I_{c}(0), I_{c}(0)=\frac{2 e}{\hbar}\left\langle\sqrt{T_{N}} \eta|\Delta|\right\rangle
$$




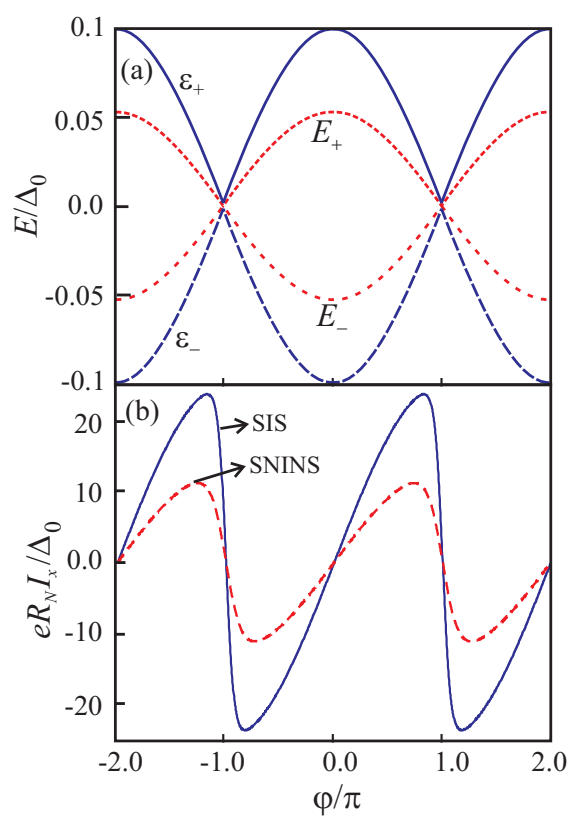

FIG. 3: (a) Energy spectrum for $n=0$ in $\mathrm{S}_{d_{x y}} \mathrm{IS}_{d_{x y}}$ and $\mathrm{S}_{d_{x y}} \mathrm{NINS}_{d_{x y}}$ junctions as a function of $\varphi$. (b) Current-phase relation for the same junctions at temperature $T=0.01 T c$.

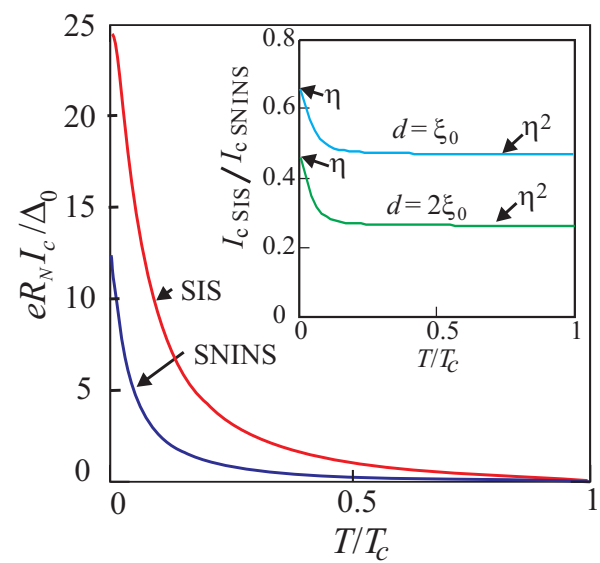

FIG. 4: Critical current for a symmetrical SNINS junction with $d=$ $2 \xi_{0}$ and an SIS junction as a function of $T$. The inset shows the ratio between the critical current of both junctions for $d=\xi_{0}$ and $d=2 \xi_{0}$. where sgn is the signum function and $I_{c}(0)$ is the critical current at $T=0$, it is proportional to $\sqrt{T_{N}} \eta$, lower than in the case of an SIS junction $(d=0)$ in a factor $\eta$. Figure $3(\mathrm{~b})$ the current-phase relation is ploted for $T=0.01 T c$, in this case the quasiparticles with energy $E_{+}$and $E_{-}$carry currents in opposite directions and the total current decreases. This is observed in Fig. 4 where $I_{C}$ is plotted as a function of temperature. When $E_{+}<<k_{B} T$ a $1 / T$ dependence current is found and $I_{c}$ is proportional to $T_{N} \eta^{2}$, lower than the critical current of an SIS junction in a factor $\eta^{2}$, this is in agreement with the results shown in the inset of Fig. 4.

\section{CONCLUSIONS}

We have found a general equation for the energy quasiparticles spectrum in SNINS junctions. With this equation we have analysed the energy spectrum in anysotropic SNS, SIS, INS and SNINS junctions. We have found analytic solutions of the general equation for energies $E<<\left|\Delta_{R / L}\right|$. For the SINS junction the energy spectrum depends on the phase difference between the two superconducting regions, the strength of the barrier and the thickness of the normal region. We have analysed several symmetries of the pair potentials; table I shows the behaviour of the energy spectrum for these symmetries. In general, we see that for $d$ symmetries we always obtain a zero energy state independent of the value of $T_{N}$. For the symmetrical SNINS junction and $d_{x y}$ symmetry of the pair potential the energy levels are modulated by an amplitude $\eta$ that depends on $d$. As a consequence the critical current in SNINS junctions is lower than the critical current of an SIS junction.

Acknowledgments The authors have received support from División de Investigaciones de la Universidad Nacional de Colombia sede Bogotá.
[1] C. C. Tsuei and J. R. Kirtley, Rev. Mod. Phys. 72, 969 (2000).

[2] A. F. Andreev, Sov. Phys. JETP 19, 1228 (1964).

[3] Y. Tanaka, S. and Kashiwaya, Phys. Rev. B 53, 9371 (1996).

[4] S. Kashiwaya, Y. Tanaka, M. Koyanagi, and K. Kajimura, Phys. Rev. B 53, 2667 (1996) .

[5] Y. Tanaka, S. Kashiwaya, Phys. Rev. Lett. 74, 3451 (1995).

[6] Y. Tanaka, S. Kashiwaya, Phys. Rev. B 56, 892 (1997).

[7] J. Bardeen and J. L. Johnson, Phys. Rev. B 5, 72 (1972).

[8] D. Rainer, J. A. Sauls, and D. Waxman, Phys. Rev. B 54, 10094 (1996).

[9] S. Kashiwaya, Y. Tanaka, M. Koyanagi, and K. Kajimura, Jpn. Appl. Phys. 34, 4555 (1995).
[10] W. Herrera and V. Niño, Phys. Stat. Sol. (b) 220, 551 (2000).

[11] A. Huck, A. Otterlo, and M. Sigrist, Phys. Rev. B 56, 14163 (1997).

[12] T. Löfwander, V. S. Shumeiko, and G. Wendin, Supercond. Sci. Technol 44, 53 (2001).

[13] Y. Tanaka, H. Tsuchiura, Y. Tanuma, and S. Kashiwaya, J. Phys. Soc. Jpn. 71 271, (2002).

[14] C. Bruder, Phys. Rev. B 41 4017, (1990).

[15] A. D. Zaikin and G. F. Zharkov, Sov. Phys. JETP 51, 364 (1980). 